\title{
ÉTUDE DE LA COAGULATION DU LAIT CONCENTRÉ
}

\author{
par \\ Charles ALAIS et Pierre STENNE \\ Institut de Chimie biologique, Faculté des Sciences, Orsay (78) \\ et Laboratoire de Recherche des Etablissements Hutin, \\ Blaise-sous-Arzillière (51). \\ (avec la collaboration technique de Jean Tasky).
}

\section{Introduction}

Au cours des essais qui ont abouti à la mise au point des nouveaux procédés “ S.H. " pour la fabrication en continu des fromages à partir de lait concentré $[1,2]$, l'un de nous (P. Stenne) a observé le comportement particulier du lait concentré, après l'action de la présure à une température relativement basse; le coagulum formé dans ces conditions présente les propriétés singulières des gels thixotropiques. Nous avons cherché à préciser les conditions de formation de ce gel en fonction de la température et dela concentration du lait ; nous présentons dans cet article le résultat d'expériences préliminaires.

Laits.

\section{Matériel et méthodes}

Le lait concentré est obtenu par traitement du lait entier pasteurisé $\left(72^{\circ}\right.$, chambrage 40 s.), dans un appareil A.P.V.-Junior (température dans les plaques de chauffe, $65^{\circ}$, détente à $45^{\circ}$ ). On prépare ainsi un lait contenant $350 \mathrm{~g}$ d'extrait sec par litre; des concentrations plus basses ont été obtenues par dilution dans l'eau distillée.

Le lait en poudre a été préparé par le procédé du brouillard (poudre Spray), après un préchauffage à $80^{\circ}$, sans chambrage.

Le phosphocaséinate naturel a été séparé par centrifugation à grande vitesse du lait de mélange écrémé, dans les conditions précédemment précisées [3] ; le sédiment a été lavé et conservé après lyophilisation.

Les protéines solubles ont été préparées par dialyse du sérum de

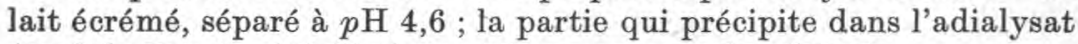
(euglobulines) a été éliminée et le surnageant lyophilisé. 


\section{Action de la présure.}

Elle a eu lieu à différentes températures, de 8 à $40^{\circ}$; la température choisie a été maintenue jusqu'à la fin des observations.

Les laits concentrés ont reçu une addition de $0,25 \mathrm{~g}$ de chlorure de calcium et de $0,3 \mathrm{ml}$ d'extrait de présure (14 U.P.) pour $90 \mathrm{~g}$ d'extrait sec dégraissé. Les solutions de laits en poudre ont reçu une addition uniforme de $0,01 \mathrm{~g}$ de chlorure de calcium et de $0,004 \mathrm{~g}$ de présure en poudre pour $100 \mathrm{ml}$ (1,8 U.P.). Un essai a été effectué avec la présure cristallisée (1,8 U.P./100 ml).

On a mesuré le temps de floculation et la vitesse de synérèse, lorsqu'elle se produit. Le moment de la floculation est malaisé à déterminer dans les laits concentrés ; le moyen le plus facile consiste à observer le film de lait qui s'écoule sur le réservoir d'un thermomètre à mercure. Le caillé est rompu et brassé 30 minutes après la floculation. La quantité de sérum séparée est mesurée après avoir jeté la masse coagulée sur un tamis métallique fin.

\section{Résultats}

Le tableau 1 schématise les observations faites à $20^{\circ}$, après l'action de la présure sur des laits entiers de différentes concentrations en extrait sec. Deux échantillons de lait ayant une concentration normale $(12,5$ p. 100) ont été traités; l'un était du lait de mélange, l'autre était obtenu par dilution du lait concentré à 35 p. 100 ; ils se sont comportés de la même manière. A cette tem.

TABLEAU 1

PROPRIÉTÉS DU COAGULUM OBTENU A PARTIR DU LAIT CONOENTRÉ A $20^{\circ}$

\begin{tabular}{|c|c|c|c|c|}
\hline & \multicolumn{4}{|c|}{ Extrait sec du lait (p. 100) } \\
\hline & 12,5 & 25 & $25(1)$ & 35 \\
\hline$p H$ du lait $\ldots \ldots \ldots \ldots \ldots \ldots$ & 6,65 & 6,45 & 6,65 & 6,35 \\
\hline Temps de floculation $(m n) \ldots \ldots$ & 110 & 39 & 55 & 31 \\
\hline Nature du coagulum (2) : & & & & $\mathrm{T}$ \\
\hline Après le 1 er brassage ( 0 h 30 ).. & $\mathrm{H}$ & $\mathrm{T}$ & $\mathbf{T}$ & $\mathbf{T}$ \\
\hline - $2^{\mathrm{e}}$ brassage $(1 \mathrm{~h} 30)$ & $\overline{\mathbf{H}}$ & $\mathrm{Th}$ & $\mathrm{Th}$ & $\mathbf{T}$ \\
\hline - $3^{\ominus}$ brassage $(2 \mathrm{~h} 30)$. & $\mathrm{C}+\mathrm{S}$ & $\mathrm{Th}$ & Th & $\mathrm{Tg}$ \\
\hline - $4^{\mathrm{e}}$ brassage $(5 \mathrm{~h} 30)$. & $\mathrm{C}+\mathrm{S}$ & $\mathrm{Th}$ & Th & $\mathrm{Tg}$ \\
\hline
\end{tabular}

(1) Lait concentré à 25 p. 100 dont le $p H$ est remonté à celui du lait frais.

(2) $H=$ masse hétérogène, granuleuse, avec du sérum.

$\mathrm{T}=$ système thixotropique; l'agitation fluidifie la masse qui se reprend ensuite en un gel ferme et homogène.

$\mathrm{Tg}=$ - id. - mais le gel formé paraît moins lisse.

$\mathrm{Th}=$ - id. - mais le gel formé paraît granuleux et "mouillé ".

$\mathrm{C}+\mathrm{S}=$ caillé séparé du sérum.

Les temps indiqués sont comptés après la floculation. 
TABLEAU II

COAGULATION DE LAITS ENRICHIS (1)

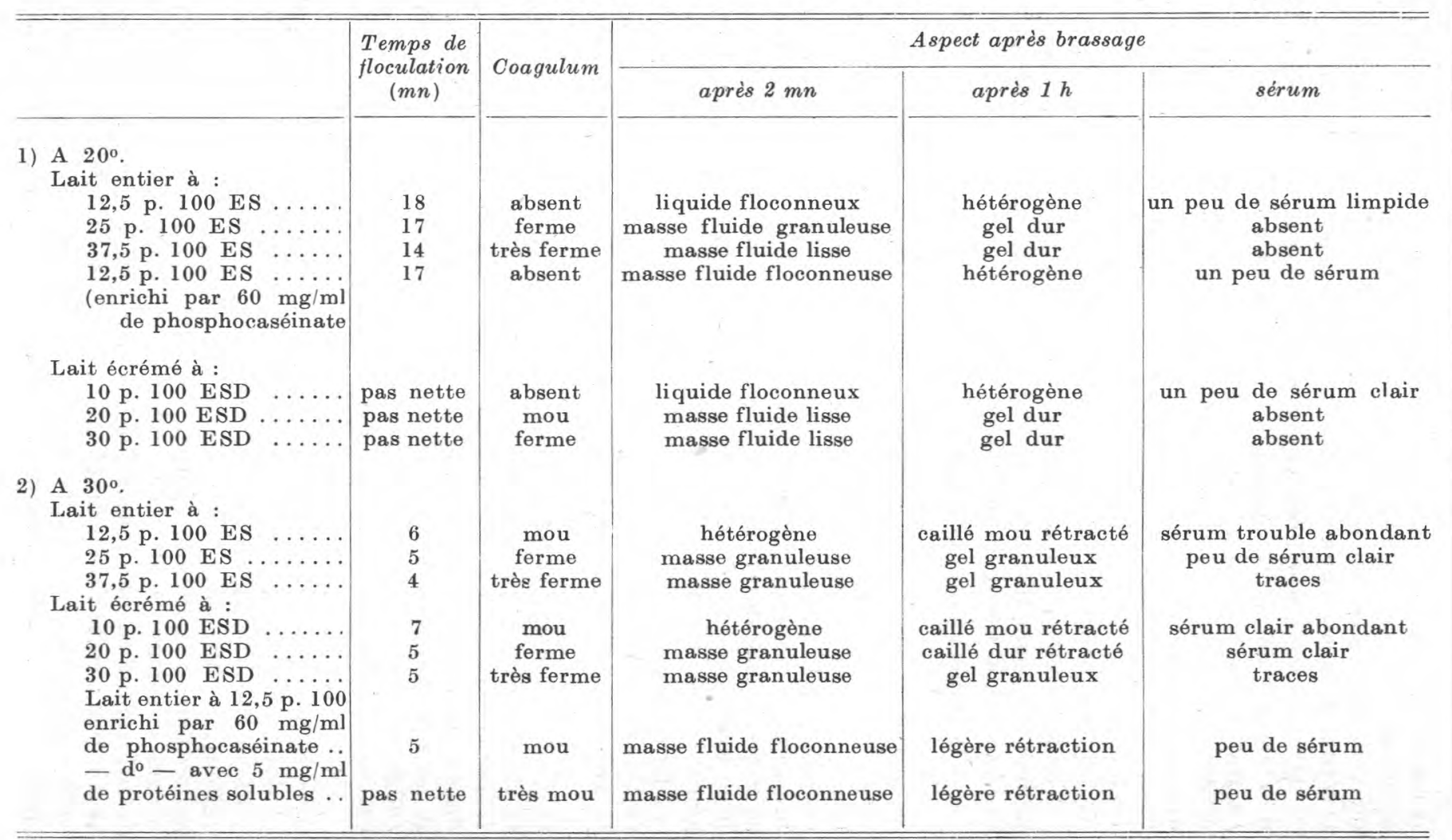

(1) Laits obtenus par dissolution de poudre de lait entier ou écrémé (voir texte). 
pérature, la coagulation et la synérèse sont très lentes ; cependant, après le premier brassage le coagulum mou devient hétérogène, on voit apparaître un peu de sérum; après le troisième brassage, le sérum se sépare et on ne peut plus le réincorporer au caillé.

Dans les mêmes conditions que précédemment, le lait à 35 p. 100 d'extrait sec donne un coagulum très ferme et qui reste homogène; l'agitation fluidifie le gel, mais celui-ci se reforme peu après; en répétant les brassages, on observe le même phénomène ; cependant, après le troisième brassage le gel qui se reforme paraît être moins lisse, un peu granuleux, mais il n'y a aucune séparation de sérum. Le lait à 25 p. 100 d'extrait sec se comporte comme le précédent au premier brassage, mais ensuite le gel paraît être " mouillé ».

Le lait concentré a un $p \mathrm{H}$ plus bas que le lait normal ; mais on n'observe aucune différence, dans la nature du coagulum, lorsqu'on ramène à 6,65 le $p \mathrm{H}$ du lait concentré.

A la température de $8^{\circ}$, le lait de concentration normale ne se gélifie pas, dans le temps de nos expériences. Par contre, le lait à 35 p. 100 d'extrait sec forme un coagulum très ferme et très lisse; un agitateur à plaque plongé dans le lait concentré avant la coagulation permet, lorsqu'elle s'est accomplie, de soulever la masse coagulée et le pot qui la contient sans briser le coagulum. Après brassage, le contenu du pot redevient immédiatement liquide; il se reprend ensuite en un gel homogène. Il semble que ce double processus puisse se répéter indéfiniment sans disparition de l'homogénéité de la masse.

Le tableau II résume les observations faites avec des laits enrichis de diverses manières, emprésurées à 20 et à $30^{\circ}$. Les propriétés thixotropiques du caillé de lait concentré tendent à disparaître à $30^{\circ}$; après brassage un gel se reforme, mais il est nettement granuleux et des traces de sérum apparaissent; l'aspect est cependant très différent de celui que présente le lait de concentration normale. Le lait écrémé se comporte comme le lait entier. Les laits mentionnés dans le tableau II ont été obtenus par dissolution de poudre de lait; il ne semble pas qu'ils présentent des propriétés différentes de celles des laits concentrés, à l'exception de la synérèse à $30^{\circ}$ qui est un peu plus marquée avec ces derniers.

Nous avons enrichi un lait entier, de concentration normale, avec du phosphocaséinate, d'une part, et avec du phosphocaséinate et des protéines solubles, d'autre part, de manière à tripler la proportion normale de ces deux constituants. Après l'action de la présure, à $20^{\circ}$, on n'observe pas de coagulation avec ces deux échantillons; le liquide épaissit et paraît floconneux; un peu de sérum se sépare. A $30^{\circ}$ nous avons observé un coagulum mou, se transformant en une masse fluide après brassage et se rétractant peu à peu ; cette forme de coagulation est donc très différente de celle du lait concentré en tous ses constituants. Mais il faut remarquer que le phosphocaséinate utilisé a été lavé avant lyophilisation 
et qu'il a été conservé à l'état sec pendant plusieurs années ; les conditions sont donc différentes de celles de l'expérience que nous avons précédemment rapportée (2).

A 30 et à $40^{\circ}$, les laits concentrés donnent un caillé qui subit la synérèse ; mais celle-ci est plus lente que dans le cas du lait normal, comme le montre le tableau III. Cependant, après 5 heures, la quantité de sérum séparée est inversement proportionnelle à la concentration du lait. A $40^{\circ}$, le rapport, sérum retenu/ES du lait, est sensiblement constant. La neutralisation du lait concentré a peu d'influence sur la synérèse. On remarque que le lait concentré, dilué avant emprésurage jusqu'à 12 p. 100 d'extrait sec, a une synérèse un peu plus lente que celle du lait normal. Si la dilution est effectuée après l'action de la présure, la quantité de sérum séparée est considérablement réduite.

\section{TABLEAU III}

SYNERESE DU CAILLÉ OBTENU A PARTIR DU LAIT NORMAL ET DU LAIT CONCENTRÉ (1)

\begin{tabular}{|c|c|c|c|c|c|c|}
\hline & \multicolumn{6}{|c|}{ Lait concentré } \\
\hline & $\begin{array}{c}\text { Lait } \\
\text { normal }\end{array}$ & $\begin{array}{l}\text { ramené } \\
\text { à l'ES } \\
\text { normal }\end{array}$ & $2 / 1$ & $\begin{array}{c}2 / 1 \\
\text { neutra- } \\
\text { lisé }\end{array}$ & $3 / 1$ & $3 / 1$ \\
\hline Extrait sec (p. 100)... & 12,5 & 12,5 & 25 & 25 & 35 & $\begin{array}{l}\text { ramené } \\
\text { à } 12,5 \\
\text { après } \\
\text { coagu- } \\
\text { lation(3) }\end{array}$ \\
\hline$\underset{\text { Sésum séparé }(2)}{p H} \cdots \cdots$ & 6,65 & 6,65 & 6,45 & 6,65 & 6,35 & \\
\hline $\begin{array}{c}\text { A } 30^{\circ} \text {, après } 1 \mathrm{~h} 30 \\
-\quad 2 \mathrm{~h} 30 .\end{array}$ & 74 & 70 & $\begin{array}{l}21 \\
45\end{array}$ & $\begin{array}{l}16 \\
45\end{array}$ & $\begin{array}{r}9 \\
25\end{array}$ & 36 \\
\hline A 40, après $\begin{array}{r}5 \mathrm{~h} 30 \\
\mathrm{~h} 30\end{array}$ & 78 & $\begin{array}{l}81 \\
70\end{array}$ & $\begin{array}{l}56 \\
44\end{array}$ & $\begin{array}{l}60 \\
39\end{array}$ & $\begin{array}{l}38 \\
32\end{array}$ & 38 \\
\hline $\begin{array}{l}2 \mathrm{~h} 30 \\
-\quad 5 \mathrm{~h} 30\end{array}$ & & $\begin{array}{l}80 \\
86\end{array}$ & $\begin{array}{l}58 \\
70\end{array}$ & $\begin{array}{l}56 \\
71\end{array}$ & $\begin{array}{l}42 \\
56\end{array}$ & \\
\hline $\begin{aligned} \text { Rapport : } & \text { sérum retenu } \\
& \text { /ES du lait }\end{aligned}$ & & & & & & \\
\hline Après 5 h 30, à $30^{\circ}$ & & 1,4 & 1,56 & 1,43 & 1,50 & \\
\hline Temps de floculation : & & 1,08 & 1,08 & 1,05 & 1,07 & \\
\hline A $30^{\circ}(\mathrm{mn}) \ldots \ldots \ldots$ & 26 & 25 & 13 & 19 & 11 & \\
\hline A $40^{\circ}(\mathrm{mn}) \ldots \ldots \ldots$ & 16 & 17 & 9 & 13 & 5,5 & \\
\hline
\end{tabular}

(1) Lait concentré (voir texte) à $3 / 1$, dilué par l'eau distillée.

(2) Volume de sérum séparé pour 100 de sérum total : $1000-(1,09 \mathrm{MG}$ $+0,74$ caséine). Les temps sont comptés après l'apparition des flocons; le découpage a lieu $30 \mathrm{mn}$ après la floculation.

(3) Le volume de sérum mesuré est diminué du volume d'eau ajouté. 
Le temps de floculation est d'autant plus court que le lait est plus concentré ; l'abaissement du $p \mathrm{H}$ résultant de la concentration n'est pas seul en cause; le lait concentré neutralisé coagule plus rapidement que le lait normal. C'est un phénomène connu [8].

Un essai de coagulation du lait entier concentré à 35 p. 100 d'extrait sec, par la présure cristallisée, a donné les mêmes résultats que la coagulation par la présure commerciale, liquide ou en poudre.

\section{Discussion}

Ces travaux préliminaires montrent que le lait concentré peut donner, après action de la présure, un coagulum ayant les propriétés d'un gel thixotropique. Ce phénomène est d'autant plus marqué que la température est plus basse et l'extrait sec plus élevé. La matière grasse ne joue aucun rôle et l'augmentation de la teneur en phosphocaséinate ne suffit pas à elle seule, à conférer les propriétés observées. Une légère variation du $p \mathrm{H}$ est sans influence.

A des températures supérieures à $20^{\circ}$, le coagulum formé par le lait concentré emprésuré tend à se comporter comme celui du lait normal; cependant, dans le premier cas la synérèse est plus lente et la proportion de sérum séparé est beaucoup plus faible que dans le second cas, mais, en définitive, le caillé retient une quantité de sérum approximativement proportionnelle à l'extrait sec du lait. La quantité d'eau liée à la paracaséine doit varier assez peu d'un caillé à l'autre, à 30 et à $40^{\circ}$; mais il faut remarquer que nos mesures ne prétendent pas à une grande précision. Cela ne signifie pas que les particules ont la même couche d'hydratation dans tous les cas. Dans le lait concentré, le diamètre moyen des particules est plus élevé que dans le lait normal [4]; lorsque le rayon de la sphère augmente, le rapport surface/volume décroît; pour une même proportion d'eau liée, la couche d'hydratation devient plus épaisse. A basse température, il est probable que la quantité d'eau liée s'accroît encore. Ces observations apportent une explication au ralentissement de la synérèse [5] ; ralentissement qui peut être tel que la synérèse paraisse inhibée. Il est à noter que, dans les procédés S.H., on ne fait pas former le caillé de lait concentré.

La thixotropie n'est pas un phénomène clairement expliqué. Une des premières théories est celle de la solvatation, basée sur l'existence d'une épaisse enveloppe de molécules d'eau orientées (lyosphère) [6]; les observations ci-dessus vont dans le sens de cette explication. Aujourd'hui on considère que la thixotropie est essentiellement une propriété des gels formés de particules anisotropiques, particules allongées autour desquelles les charges électriques et les molécules d'eau sont inégalement réparties. Les particules de caséine native, dans le lait normal, paraissent sphé. 
riques; il n'en va probablement pas de même dans le lait concentré, car il a été montré que les particules se déforment au cours de la concentration [4]; il est done possible qu'elles présentent l'anisotropie.

L'étude des sols minéraux ou organiques a montré qu'il existe une condition essentielle à la formation du gel thixotropique : une concentration optimale en électrolytes. En-dessous d'une certaine concentration saline, le sol est stable; mais au-dessus d'un autre seuil de concentration, la floculation se produit [7]. De plus, la concentration de la substance colloïdale doit se trouver entre certaines limites. Il est probable que la teneur en sels et la teneur en phosphocaséinate jouent un rôle dans le phénomène que nous avons observé; mais le système n'est pas identique aux sols précédents. Ce système est plus complexe, deux actions sont superposées, la concentration en électrolytes et en particules, d'une part, et l'action enzymatique, d'autre part. On peut faire l'hypothèse de l'existence de deux types de coagulation :

1) Gélification ayant un caractère réversible, consistant dans la formation d'une structure faible, par suite de l'orientation réciproque de particules anisotropiques. Elle serait favorisée par des températures relativement basses et dépendrait de la concentration en extrait sec.

2) Coagulation irréversible telle qu'elle se produit dans les conditions habituelles de l'action de la présure sur le lait normal, à température moyenne. Il s'agit là d'une structure plus résistante, résultant de l'établissement de liaisons chimiques et le gelformé subit la synérèse.

Le premier type de coagulation est purement physique; e'est un phénomène thixotropique simple. On connaît la gélification spontanée des laits concentrés; mais celle-ci ne survient qu'après un temps que l'on compte en jours ou en mois. Il faut donc admettre que l'action de la présure est nécessaire à la gélification réversible, comme à la coagulation proprement dite.

Si les modifications que subissent les constituants du lait, au cours du traitement thermique appliqué au lait concentré ou au lait desséché, jouaient un rôle dans les phénomènes observés, il faudrait admettre qu'il ne peut se manifester qu'en milieu suffisamment concentré ; puisque le gel thixotropique ne se forme pas, à $20^{\circ}$, à partir du lait concentré ramené à 12,5 p. 100 d'extrait sec.

Il y a encore des points obscurs; nous poursuivons nos travaux dans le but de déterminer l'influence de la concentration saline et l'influence du chauffage et de rechercher si un gel thixotropique peut se produire en dehors de l'action de la présure. 


\section{BIBLIOGRAPHIE}

[1] P. Stenne, Le Lait, 1965, [45], nos 441-2 et 443-4.

[2] C. AlaIs, Industrie Laitière. 1965, no 218, 90.

[3] G. Mocquot, C. Alais et R. Chevalier. Ann. Technol. Agric., 3, 1954, 1,

[4] H. Hostettlen. Schweizerische Milchzeitung, $\mathrm{n}^{\circ} 82,1951,531$.

[5] C. L. Hankinson et L. S. Palmer, J. Dairy Sci., 26, 1943, 1063.

[6] S. Glasstone. Textbook of Physical Chemistry, London, 1951.

[7] J. Duclaux. Traité de Chimie Physique appliqué à la Biologie, Paris, 1934.

[8] S. Odagiri et T. A. Nickerson. J. Dairy Sci., 47, 1964, 1306.

\section{Résumé}

On observe la formation d'un gel thixotropique lorsque l'on fait réagir la présure sur le lait concentré à température moyenne et à basse température. La matière grasse ne joue aucun rôle dans ce phénomène et une teneur élevée en phosphocaséinate ne suffit pas à le provoquer. La quantité de sérum retenu par le caillé, dans les conditions où la synérèse se produit, est proportionnelle à l'extrait sec du lait.

\section{Summary}

When rennet is added to a concentrated milk (3/1) at temperatures of $20^{\circ} \mathrm{C}$ and lower, it may be observed the formation of a curd in a thixotropic form. The fat does not act on this phenomenon no more than a higher proportion in phosphocaseinate. The percentage of whey which is retained by the curd after cutting, when syneresis occurs, is proportionnal to the total solids of rennetted milk. 